JLAB-THY-01-33

\title{
Topology and Low Lying Fermion Modes
}

\author{
Robert G. Edwards ${ }^{\mathrm{a}}$ \\ a Jefferson Lab \\ 12000 Jefferson Avenue \\ MS 12H2, Newport News, Virginia, USA \\ Recent results concerning the relation of topology and low-lying fermion modes are summarized.
}

\section{Introduction}

Low lying fermionic modes are believed to play an important rôle in QCD. This review covers some of the recent developments in the study of the relationship of low lying fermion modes with topology in QCD. The study of topology in general is not covered here. In particular, fermionic eigenmodes (including zero modes) are important in 3 areas discussed here: (1) low lying modes in particle spectrum, (2) low lying modes in global topology (e.g., chiral fermions), (3) low lying modes in the implementation of chiral fermions. The use of fermionic modes to probe for possible mechanism of chiral symmetry breaking in QCD are possible. The recent advent of the construction of chiral fermions on the lattice are crucial in new studies.

\section{Spectrum}

\subsection{Eigenmodes in Spectrum}

One expects low lying fermionic eigenmodes to be important in quark propagation because they should dominate the spectral sum for small enough quark masses. By computing some number of the lowest lying eigenvectors of the Dirac operator one can expect that most of the physics of the quark propagator can be obtained. To make these statements more precise, an example is given of the computation of the $\eta^{\prime}$ mass. Consider the spectral decomposition of a quark propagator - for simplicity use a hermitian form of the Dirac operator

$$
H=\gamma_{5} D_{w}, \quad H \psi_{i}(x)=\lambda_{i} \psi_{i}(x)
$$

where $D_{w}$ is the standard Wilson-Dirac operator, $H$ is the hermitian Wilson-Dirac operator and the $\psi_{i}(x)$ are the eigenvectors with eigenvalue $\lambda_{i}$. Then the spectral representation of the quark propagator is

$H^{-1}(x, y)=\sum_{i} \frac{\psi_{i}(x) \psi_{i}^{\dagger}(y)}{\lambda_{i}}$.

The correlation function for the $\eta^{\prime}$ involves a disconnected piece which is typically stochastically estimated. A possible improvement is to truncate the spectral sum with the lowest few eigenvectors (which should give the largest contribution) and stochastically estimate the remainder. The idea is to represent $H=\sum_{i} H_{i}+H_{\perp}$ where $H_{\perp}$ are the remaining modes. Then in all relations where $H^{-1}$ enters, find all the terms involving only $\sum_{i} H_{i}$ and the cross terms involving $H_{\perp}$. Since the eigenvectors contain the full information (in the subspace) of the propagator (not just emanating from a single point), impose translation symmetry by summing across the lattice for the lowest modes. This sum gives a volume times more statistics for the modes (hopefully) dominating the correlation function. For the remaining terms involving $H_{\perp}^{-1}$ use a stochastic estimator. The linear system solutions involved in this latter step are accelerated since the condition number is lowered from projecting out the lowest eigenmodes.

How many eigenmodes are needed? The scale should be set by the chiral condensate, hence the number should grow at least like the volume.

Instead of computing the lowest eigenmodes, deflation can be used to compute the eigenvectors at the time of the linear system solution thus 


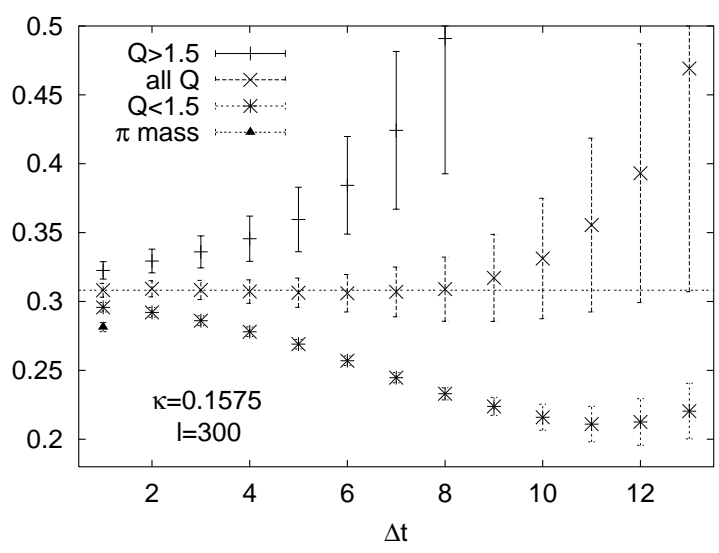

Figure 1. Dependence of $\eta^{\prime}$ effective mass on topological charge (Ref. [2]).

accelerating the stochastic estimation [1].

One obvious question is whether to use for Wilson-like fermions the hermitian or nonhermitian operator. A comparison of the truncated spectral sum of the pion propagator shows poor convergence in the non-hermitian version when adding more eigenvectors [2]. The hermitian version shows slow but even convergence. For chiral fermions, the choice is irrelevant.

The Wuppertal group has used the truncated spectral version of the hermitian quark propagator for a calculation of the $\eta^{\prime}$ correlation function using $N_{f}=2$ dynamical Wilson fermions [2]. A similar calculation was made for staggered fermions [3]. One goal is to determine the topological charge dependence of the $\eta^{\prime}$ mass. Using a fermionic definition of the charge and binning configurations according to $Q$, the effective masses show a $Q$ dependence as seen in Fig. 1. They find the truncated spectral sum method competitive with stochastic estimation for their large quark masses. The spectral sum method will not suffer dramatically going to smaller quark masses, however. The truncation of the spectral sum is evident in the not very cosh-like behavior of the $\eta^{\prime}$ correlation function. The calculation could be made exact by including the extra stochastic estimation.

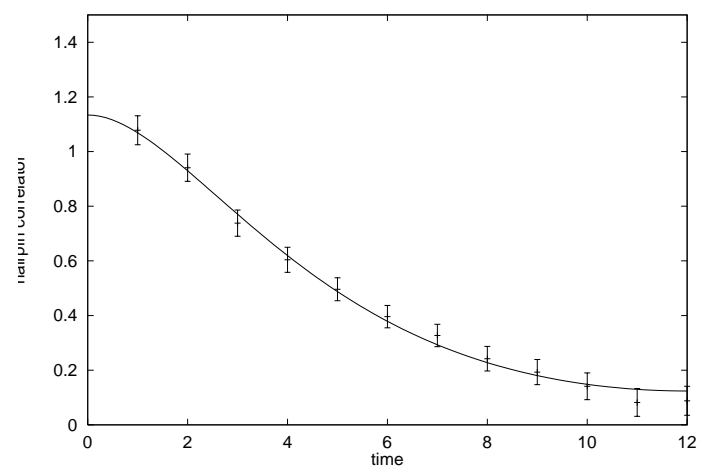

Figure 2. One-parameter fit to a pure doubleGoldstone pole form for the $\eta^{\prime}$ hairpin correlator. The pion mass is fixed from the valence propagator analysis (Ref.|15]).

While not using a fermionic method, a calculation by the UKQCD collaboration of the flavor singlet mesons with $N_{f}=2$ dynamical $\mathcal{O}(a)$ improved Wilson fermions has been made [4]. While statistically noisy, the OZI rule of suppressed singlet - non-singlet mass splittings is seen like in the quenched case $[5]$.

\section{Topology}

\subsection{Topological Susceptibility}

The topological susceptibility is intimately tied with low lying fermion modes and is an important probe of the QCD vacuum relating to the $U_{A}(1)$ problem. Pure gauge calculations of the topological susceptibility are consistent with the WittenVeneziano prediction [6. Another important test is to determine how the susceptibility depends on the quark mass. There are several recent large calculations of the topological susceptibility using $N_{f}=2$ dynamical fermions, from CPPACS (mean-field clover) [7], UKQCD (non-pertubative clover) [8], SESAM $/ \mathrm{T} \chi \mathrm{L}$ (Wilson) [9], and thinlinked staggered [10]. By continuum arguments, the susceptibility is expected to behave like

$\chi(m)=\frac{\Sigma m}{N_{f}}(1+\mathcal{O}(m))=\frac{m_{\pi}^{2} F_{\pi}^{2}}{2 N_{f}}(1+\mathcal{O}(m)) .(3)$

Only the UKQCD calculation claims to unambiguously see the expected decrease of $\chi(m)$ 


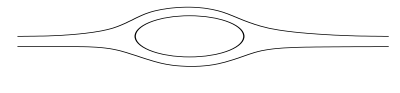

(a)

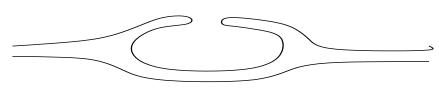

(b)

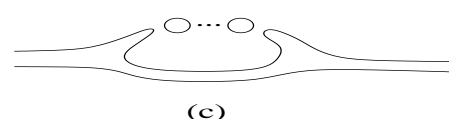

Figure 3. Contributions to the $a_{0}$ propagator from an $\eta-\pi$ intermediate state.

by lowering $m$. A comparison of the various results at finite lattice spacing requires taking into account discretization errors [11. Naïve linear extrapolations in $m_{\pi}^{2}$ (fixing $F_{\pi}$ ) give poor fits, suggesting that discretization effects are large. Besides large quark masses used there are other sources of systematic errors including the definition of $\chi(m)$ which involves contact terms and mixing with the unit operator [6]. For a chiral fermion action with a fermionic definition of $\chi(m)$ these counter-terms are absent only in the $N_{f}=2$ chiral limit. They could be avoided by using a finite-volume scaling technique 12 for small $m$ applied to $\chi(m)$.

\subsection{Quenched Pathologies}

Another area low lying eigenmodes play an important role are in quenched pathologies. How well is QCD described by an effective chiral theory of interacting particles (e.g., pions in chiral dynamics)? Suppressing the fermion determinant leads to well known pathologies as studied in chiral perturbation theory [13]. The pathologies are manifested in the $\eta^{\prime}$ propagator missing vacuum contributions. A new dimensionful parameter is induced in $\chi \mathrm{PT}$. Power counting rules are changed leading to new chiral logs and powers terms. The predicted pathologies for the pion mass were studied with Wilson fermions by CPPACS [14]. There are recent studies with Wilson fermions in the Modified Quenched Approximation [15,16] and using the overlap chiral fermion

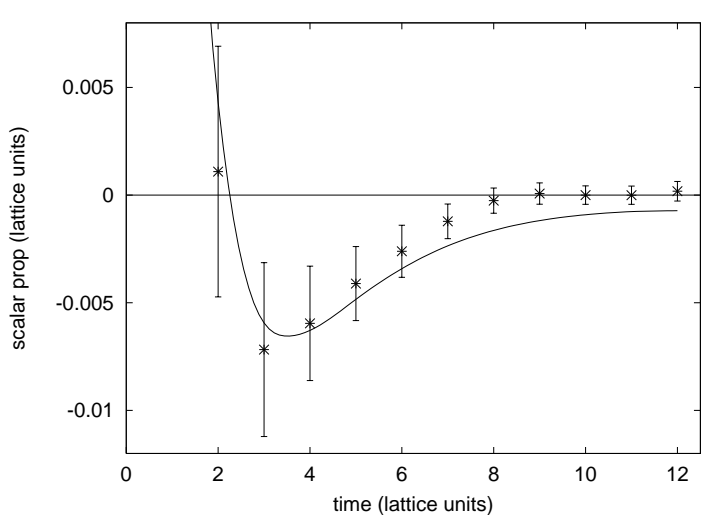

Figure 4. Comparison of scalar $a_{0}$ propagator with the bubble sum formula fitted to the interval $\mathrm{t}=1-6$ (Ref. 16]).

operator [17].

The pion mass was one of the first observables studied for the effects of quenched chiral logs; however, it is not necessarily the easiest place to look. The additional quenched logs appear in the pion mass from the missing loop contributions in the $\eta^{\prime}$ correlator. In [15], the unique piece of the $\eta^{\prime}$ correlator - the hairpin - was computed directly and compared to quenched $\chi \mathrm{PT}$. Fig. 2 shows the correlator along with the fit holding $m_{\pi}$ fixed to the simple mass insertion formula given by

$$
\begin{gathered}
\int \frac{d^{4} p}{(2 \pi)^{4}} e^{i p \cdot x}\left\langle\operatorname{Tr} \gamma_{5} G(x, x) \operatorname{Tr} \gamma_{5} G(0,0)\right\rangle= \\
f_{P} \frac{1}{p^{2}+m_{\pi}^{2}} m_{0}^{2} \frac{1}{p^{2}+m_{\pi}^{2}} f_{p}
\end{gathered}
$$

The correlator is well described by this form with the only free parameter the $\eta^{\prime}$ mass insertion $m_{0}^{2}$. The pseudoscalar decay constant also shows the expected quenched artifact given by

$f_{p}^{\text {quenched }}=\left(\frac{1}{m_{\pi}^{2}}\right)^{\delta} \tilde{f}_{P}$

while the axial vector decay constant does not show such a divergence. After considering various observables, the overall determination of $\delta=$ 0.065 (13) compared to $\delta=0.18$ in Ref. 13 .

In Ref. [17, the overlap-Dirac operator was computed on a $20^{4}$ lattice at a lattice spacing of 
$a=0.13 \mathrm{fm}$. Quenched chiral logs were observed in $m_{\pi}^{2}$ and $f_{P}$ giving $\delta \sim 0.15$ to 0.4 .

A dramatic example of quenching is exhibited in the non-singlet scalar particle 16]. For the $a_{0}$, there is an $a_{0} \rightarrow \eta-\pi$ intermediate state as seen in Fig. 3. The vacuum contribution (a) is missing in the quenched approximation along with the vacuum bubbles on the hairpin piece (c), but a remaining hairpin piece (b) is present. The remaining hairpin piece has a quadratic divergence coming from the three pion propagators present. This quenched artifact causes a loss of positivity of the propagator as seen in Fig. 4 . The fit is to the 1-loop bubble term as computed in quenched $\chi \mathrm{PT}$ and is in good agreement with the data 16]. The difficulty in this Wilson fermion (MQA) approach is the mixing of would-be zero modes and non-zero modes and in particular what are the contributions from each part. For example, is the lack of positivity a zero mode effect? It is not expected to be, but more will be discussed on zero modes in the next section.

While the $a_{0}$ quenched artifact can be viewed as the remnants of a decay, a true decay requires full QCD. The MILC collaboration has used an improved staggered fermion action in a $N_{f}=2+1$ calculation of the $a_{0}$ decay [18]. Evidence is found for a level splitting in the $a_{0}$ mass and an $\eta+\pi$ state.

\subsection{Condensate}

Several model calculations indicate the quenched chiral condensate diverges at $T=0$ (e.g., Ref. 19]). Damgaard [20], shows via quenched $\chi \mathrm{PT}$ that the first finite volume correction to the chiral condensate diverges logarithmically in the 4-volume. Some simple relations for susceptibilities of pseudoscalar and scalar fields can be derived in the continuum and on the lattice with a chiral fermion action (see for example Ref.[21). Define $\pi$ and $a_{0}$ fields by

$\pi^{a}(x)=i \bar{\psi}(x) \gamma_{5} \tau^{a} \psi(x), a_{0}(x)=-\bar{\psi}(x) \tau^{a} \psi(x) .(6)$

Then the $\pi$ and $a_{0}$ susceptibilities are given by

$$
\begin{aligned}
\sum_{x}\left\langle\pi^{a}(x) \pi^{a}(0)\right\rangle & =\frac{1}{m}\langle\bar{\psi} \psi\rangle \\
\sum_{x}\left\langle a_{0}^{a}(x) a_{0}^{a}(0)\right\rangle & =\frac{1}{m}\left\langle\frac{d}{d m}\langle\bar{\psi} \psi\rangle_{A}\right\rangle .
\end{aligned}
$$

Note in the quenched approximation the $a_{0}$ susceptibility is given by the derivative of the chiral condensate. These relations are true including and excluding global topology terms.

The Banks-Casher relation on a finite lattice is

$$
\frac{1}{V} \sum_{x}\langle\bar{\psi}(x) \psi(x)\rangle=\frac{|Q|}{m V}+\frac{1}{V} \sum_{n} f\left(\lambda_{n}, m\right),
$$

where $f$ is some unspecified function of the nonzero eigenvalues. One sees the global topology term is irrelevant in the thermodynamic limit. Taking the appropriate limits the usual relation is recovered

$$
\lim _{m \rightarrow 0} \lim _{V \rightarrow \infty} \frac{1}{V} \sum_{x}\langle\bar{\psi}(x) \psi(x)\rangle=\pi \rho\left(0^{+}\right) .
$$

If the chiral condensate diverges without the topology contribution, the $a_{0}$ susceptibility must be negative and diverge. This divergence requires large enough physical volume to be apparent. Difficulties in the past include: (1) mixing of (would-be) zero and non-zero modes with staggered fermions, (2) mixing of topology and non-zero modes and contact terms with Wilson fermions, (3) until recently chiral fermion studies not on large enough lattices, e.g., random matrix model tests, spectrum tests, direct measurement tests. More direct tests of the divergence of the condensate will come from thermodynamics.

\subsection{Thermodynamics}

The deconfined phase of $\mathrm{SU}(2)$ quenched gauge theory, $L^{3} \times 4, \beta=2.4$, above $N_{t}=4$ transition was studied using the overlap-Dirac operator [22]. A study of the build-up of the density of eigenvalues near zero, $\rho(E)$ indicates the quenched chiral condensate (without topology) is diverging [22]. Define the density of zero eigenvalues $\rho(E)$ from the derivative of the cumulative distribution

$$
\begin{aligned}
N(E, V) & =\#(\lambda>0) \text { where } \lambda<E \\
\rho(E) & =\frac{d}{d E} \lim _{V \rightarrow \infty} \frac{N(E, V)}{V} .
\end{aligned}
$$

As seen in Fig. 5, the cumulative distribution appears to continually rise and track line on a log plot - hence the derivative (the condensate) diverges with increasing lattice size. 


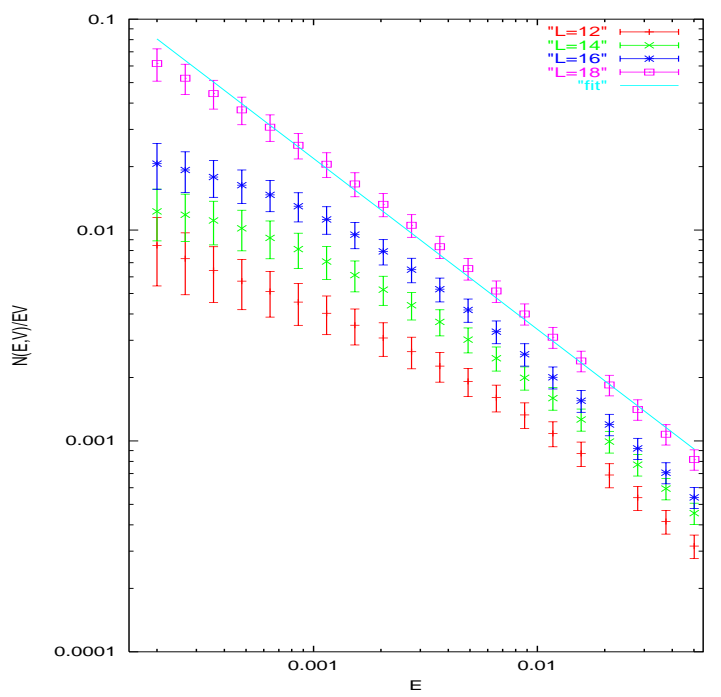

Figure 5. A plot of $N(E, V) /(E V)$ from the small nonzero eigenvalues on four different lattices plotted on a log-log scale. The solid line is a least squares fit to the $L=18$ data weighted by the statistical errors (Ref.[22]).

A diverging condensate indicates the spectral gap is closed. However, a decrease in the topological susceptibility is seen when crossing to $T>0$. Has the quenched approximation distorted the vacuum so much that it has invalidated continuum arguments as to the nature of the $T>0$ phase transition or mechanisms of confinement? The claim is no - there is merely a large accumulation of near zero fermion modes. However, models predict changes in the vacuum structure crossing to the deconfined and (supposedly) chirally restored phase and low lying fermion modes can again be used to probe the vacuum.

\subsection{QCD Vacuum}

It is generally accepted that the QCD vacuum is characterized by strongly fluctuating gluon fields with clustered or lumpy distribution of topological charge and action density. Confinement mechanisms are typically ascribed to a dualMeissner effect - condensation of singular gauge configurations such as monopoles or vortices (see review Ref. [23]). Disagreements of various mod- els include: (1) instanton models provide chiral symmetry breaking, but not confinement, (2) center vortices provide confinement and chiral symmetry breaking, (3) composite nature of instantons (linked by monopoles - calorons) at $T>0$. A different approach relying on properties of gauge field correlators and not specific gauge field types describes a large class of non-perturbative phenomena 24].

Singular gauge fields are probably intrinsic to $\mathrm{SU}(3)$ (e.g., not just Gribov copies associated with gauge fixing). These singularities impose boundary conditions on quark and gluon fluctuations which moderates the QCD action. For example, instantons have locked chromo-electric and magnetic fields $E^{a}= \pm B^{a}$ that decrease in strength in a certain way.

In a hot configuration one expects huge contributions to the action beyond such special type of field configurations. However, there could possibly be domains of (near) field locking. In recent calculations 25], these domains have been shown to be sufficient to produce chiral symmetry breaking, and confinement (area law).

\subsection{Instanton Dominance in QCD?}

Witten has argued [26] that topological charge fluctuations are clearly involved in solving the $U_{A}(1)$ problem. However, the dynamics of the $\eta^{\prime}$ mass generation need not be associated with semiclassical tunneling events. In particular, large vacuum fluctuations from confinement also produce topological fluctuations. Large $N_{c}$ arguments are incompatible with instanton based phenomology - namely instantons produce and $\eta^{\prime}$ mass that vanishes exponentially, but from large $N_{c}$ chiral dynamics suggest that $m_{\eta^{\prime}}^{2} \sim 1 / N_{c}$. Witten speculated that the $\eta^{\prime}$ mass comes from the coupling of $U_{A}(1)$ anomaly to topological charge fluctuations and not instantons.

\subsection{Local Chirality}

A test of Witten's conjecture was put forward with a local measure of chirality of non-zero modes [27]. The relative orientation of the left and right handed components of low lying eigen- 

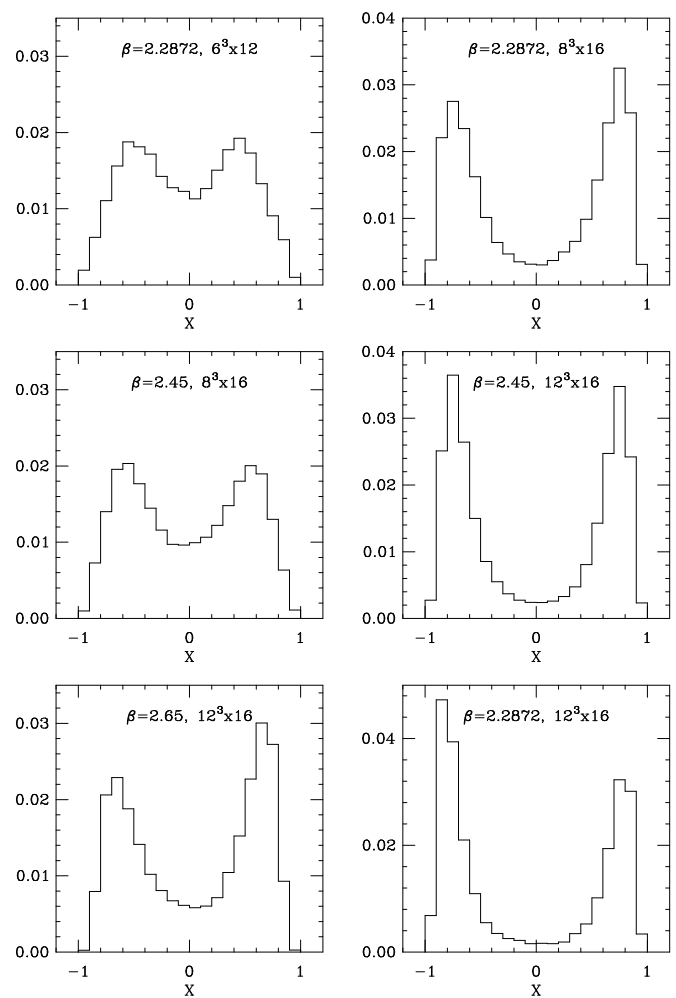

Figure 6. Chirality histograms for the lowest two non-zero modes of the overlap-Dirac operator at the $2.5 \%$ sites with the largest $\psi^{\dagger} \psi(x)$ on the six ensembles with Iwasaki gauge action. The systems in the left column all have approximately the same volume in physical units. The systems in the top two panels in the right column also have the same, about a factor of 3 larger, volume, while the system in the lower right hand corner panel has the largest volume (Ref. [29]).

modes is used

$\tan \left(\frac{\pi}{4}(1+X(x))\right)=\sqrt{\frac{\psi_{L}^{\dagger}(x) \psi_{L}(x)}{\psi_{R}^{\dagger}(x) \psi_{R}(x)}}$.

Here $X(x)$ is a site dependent measure of the local chirality. A completely chiral state (an exact zero mode) have for some sites $X(x)= \pm 1$. These regions of local chirality come from (near) locking of the chromo-electic and magnetic $(E$ and $B)$ fields. Wilson fermions were used as a test 27], and since the chirality appeared random the claim was there is no instanton dominance in the QCD

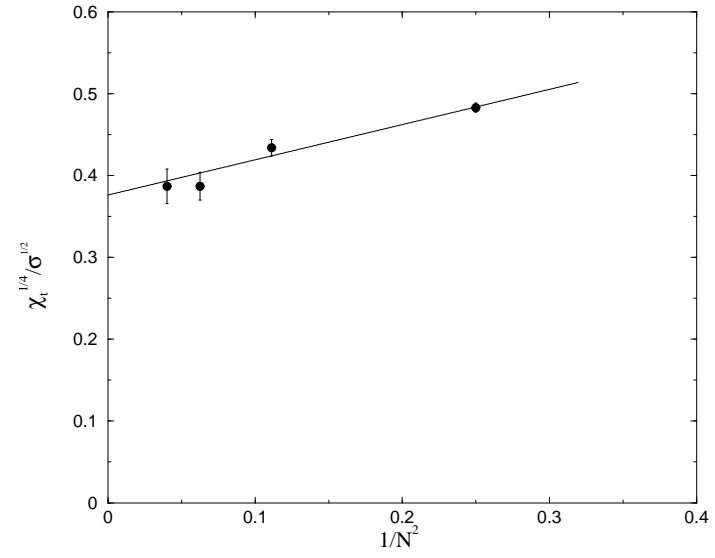

Figure 7. The continuum topological susceptibility in units of the string tension plotted against $1 / N^{2}$. A linear extrapolation to $N=\infty$ is shown (Ref. [31]).

vacuum. A flurry of papers using improved improved actions followed 28,29.

The chirality histogram for the overlap-Dirac operator is shown in Fig. 6. Shown is the histogram of $X(x)$ for $2.5 \%$ of the sites with the largest $\bar{\psi} \psi$ at three physical volumes keeping only the lowest two non-zero modes. Keeping more small non-zero modes indicates a finite density of such chiral peaked modes which may survive the continuum limit 29]. The mixing (trough) in the histograms are not related to dislocations. Namely, comparing histograms from different gauge actions and vastly different gauge field dislocation content appear identical indicating mixing comes from large sized eigenmodes. In addition, no significant peaking was found in a study of $U(1)$ in the confined phase where instantons are not present [30].

These results are all consistent with instanton phenomology; however, more generally they are consistent with suitable regions of (nearly) locked E \& B fields.

\subsection{Large $N_{c}$}

Why is there a concern of reconciling the large $N_{c}$ limit of $\mathrm{QCD}$ with studies of the QCD vacuum? The large $N_{c}$ description of QCD is quite 
successful phenomenologically. It forms a basis for the valence quark model and OZI rule, and well describes hadron spectra and matrix elements. The Witten-Veneziano prediction for relating the topological susceptibility and meson masses may well be reached at $N_{c}=3$.

How do gauge theories approach the limit? The prediction is that for a smooth limit, one should keep a constant t'Hooft coupling, $\lambda=g^{2} N$ as $N_{c} \rightarrow \infty$. Is the limit realized quickly? Fig. 1 shows a test for the quenched gluonic topological susceptibility [31]. The large $N_{c}$ limit is apparently realized quickly (seen more definitely in a $2+1$ study [32]) and is consistent with the predicted $1 / N_{c}^{2}$ scaling [31].

Revisiting the issue of local chirality, it was found that the amount of peaking observed for example in Fig. 6 decreases at a fixed scale set by the string-tension as $N_{c}$ increases from 3 to 4 [33]. This disappearance of local chirality is consistent with large (instanton-like) modes disappearing. In fact, Witten 26] predicts a strong exponential suppression of instanton number density. Teper 34] argued that including combinatorial factors for the measure could compensate for the exponential suppression leading to a sharp cutoff of the size distribution for small modes, but non-zero above the cutoff. However, given this limited step in increasing $N_{c}$ the decrease in local chirality is consistent with the large $N_{c}$ predictions. More work is needed to sort out contributions to the size distribution from dislocations. In any case, the large $N_{c}$ limit is an interesting place to gain additional understanding of the QCD vacuum. Chiral fermions have been essential in these studies.

\subsection{Eigenmode Dominance}

How much are hadron correlators dominated by low eigenmodes? In recent work [35,36] comparisons were made of the truncated and full spectral decomposition of various correlation functions using the (APE smeared) overlap-Dirac operator. The lowest 20 modes (including zero modes) were computed. These new results with a chiral fermion action draw some of the same conclusions as older works 3.37. Namely, for small quark masses the non-singlet pseudoscalar corre- lator (pion) is well approximated by the truncated spectral sum. The non-singlet vector correlator only saturates at long time distances. These results are consistent with instanton phenomology.

The QCD sum rule approach parameterizes short distance correlators via the operator product expansion and long distance by condensates. There are large non-pertubative physics in nonsinglet pseudoscalar and scalar channels. With the overlap-Dirac operator above [36], the truncated spectral sum for the point-point propagators shows the appropriate attractive channel for the pseudoscalar and repulsive channel for the vector predicted by instanton phenomology and consistent with the Wilson case [37].

$R_{i}(x)=\Pi_{i}(x) / \Pi_{i}^{0}(x), \quad \Pi_{i}(x)=\operatorname{Tr}\left\langle J_{i}^{a}(x) J_{i}^{a}(0)\right\rangle$, $J_{i}^{a}(x)=\bar{\psi}(x) \tau^{a} \Gamma(i) \psi(x)$

Fig. 8 shows the ratio $R_{i}(x)$ for the full and truncated pseudoscalar case with the free propagator for different quark masses. Saturation requires few modes for the lightest masses. One caveat is the use of APE smearing for the gauge links which could adversely affect the short distance part especially for heavier masses.

\subsection{Screening Correlators}

In the high temperature phase of $\mathrm{QCD}$, one expects restoration of chiral symmetry exhibited in the limit $m_{q} a \rightarrow 0$ by the equivalence of (nonsinglet) screening correlators

$C_{S}(z)=-C_{P S}(z), \quad C_{V}(z)=C_{A V}(z)$

summed over the dimensions orthogonal to the $z$ direction. A quenched calculation [38] with the overlap-Dirac operator at the $N_{t}=4$ transition shows good agreement in the vector $(\mathrm{V})$ and axial-vector (AV) channels whereas previous $N_{f}=0 \& 2$ calculations did not show agreement in the scalar (S) and pseudoscalar (PS) channels. The zero mode contributions tend to spoil Eq. 13 but are a finite volume artifact. One can subtract them out directly or cancel them by comparing $\left(C_{S}(Z)-C_{P S}(z)\right) / 2$ to $C_{P S}(z)$ without zeromodes. The agreement without the zero modes tends to support the conclusion that chiral symmetry is restored and parity doubling is seen. However, the lack of a diverging chiral condensate 

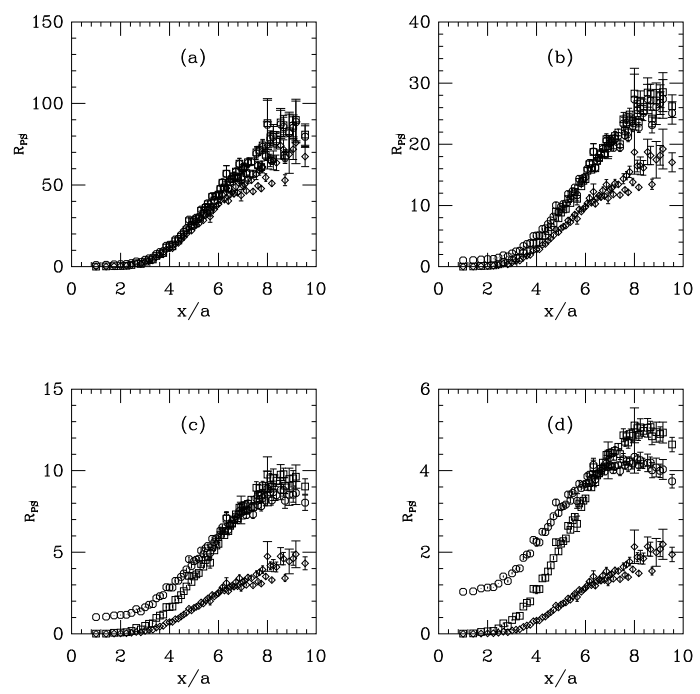

Figure 8. Saturation of the point-to-point pseudoscalar correlator by low-lying eigenmodes of the hermitian overlap operator. Results for 4 quark masses are shown with $m_{P S} / m_{V}$ ranging from 0.34 in (a) to 0.64 in (d). Octagons show the full hadron correlator. Squares show the contribution from the lowest 10 modes. Diamonds show the contribution from the zero modes, which just scales as $1 /\left(a m_{q}\right)^{2}$ (Ref. [36]).

seen in Ref. 22 probably from a small physical spatial volume indicates that increasing the size could break the parity doubling once again. More work to resolve this issue is needed.

\subsection{Localization}

With chiral fermions, one can test the issue of localization of fermionic eigenmodes and can possibly compare to localization of gluonic quantities. The latter is however strongly affected by dislocations in the gauge fields forcing the need for cooling or smearing. This unfortunately can affect part of what one was hoping to test. However, fermionic studies [35, 36, 38, 40 provide evidence for localization.

\section{Chiral Fermions}

While low eigenmodes of chiral fermions have been discussed, the implementation of the chiral fermion operators is strongly affected by low lying modes. For recent reviews of the over- lap and domain wall (DWF) constructions see Refs. 41.42]. In particular, the five-dimensional domain wall operator is equivalent to the fourdimensional overlap after taking the extent of the fifth dimensional to infinity. The overlap operator has the form $D_{\text {overlap }}(0)=\left(1+\gamma_{5} \varepsilon(H(-M))\right) / 2$ where $H(-M)$ could be the super-critical hermitian Wilson-Dirac operator with mass $M$. The numerical implementation of $\varepsilon(H(-M))$ whether by an extra dimension via DWF or some rational approximation is adversely affected by small eigenvalues of $H(-M)$. The deviation of $\varepsilon(H(-M))$ from \pm 1 induces chiral symmetry breaking 29,43]. In both the overlap and DWF case one can project out some number of low lying eigenmodes and correct the $\varepsilon(H(-M))$ to \pm 1 [44].

The issue of whether there is a large number or even a finite density of small (zero) eigenvalues of $H(-M)$ is a technical problem related to Wilsonlike gauge actions since it can be shown that given bounds on a plaquette from unity there is also a corresponding bound on $\lambda_{\min }(H(-M))$ 45]. There are classes of configurations not satisfying these bounds that induce exponentially localized zero-modes of $H(-M)$ which occur with non-zero density in a Wilson-like gauge action, and that have been observed to rapidly decrease with coupling [46]. The particularly troublesome modes are from dislocations of the gauge fields. The density of these modes is greatly affected by changing the gauge action and lowering plaquette fluctuations 447. In practice, the effects on $\varepsilon(H(-M))$ can be fixed by projection or using a weaker coupling or both making the question of a finite density somewhat moot. However, for a dynamical calculation in the chiral limit the zero level crossings of $H(-M)$ should be repelled from crossing at $M$ of the simulation, hence no ambiguity in the construction of a chiral fermion.

At very strong coupling a new phenomena emerges where the density of the dislocations becomes so large that the single flavor region in $M$ merges with the doubler region $(M>2)$. Strong coupling and mean-field calculations differ over this mixing [48], and the approach to the continuum limit. 


\section{Conclusions}

Low lying fermionic modes provide a powerful probe of the vacuum. There are many studies using fermionic modes in quenched theories. Obviously more studies are needed with dynamical fermions.

This work was supported by DOE contract DEAC05-84ER40150 under which the Southeastern Universities Research Association (SURA) operates the Thomas Jefferson National Accelerator Facility (TJNAF).

\section{REFERENCES}

1. R. Morgan and W. Wilcox, hep-lat/0109009.

2. H. Neff, et.al., hep-lat/0106016.

3. L. Venkataraman and G. Kilcup, heplat/9711006

4. C. McNeile, et.al., hep-lat/0107003.

5. N. Isgur and H. Thacker, Phys. Rev. D64 (2001) 094507.

6. L. Giusti, et.al., hep-lat/0108009.

7. A. Ali Khan, et.al., Phys. Rev. D64 (2001) 114501.

8. A. Hart, et.al., hep-lat/0108006.

9. G. Bali, et.al., Phys. Rev. D64 (2001) 054502.

10. B. Alles, et.al., Phys. Lett. B483 (2000) 139. A. Hasenfratz, Phys. Rev. D64 (2001) 074503.

11. S. Durr, hep-lat/0108015.

12. P. Damgaard, et.al., Phys. Rev. D61 (2000) 094503.

13. C. Bernard and M. Golterman, Phys. Rev. D46 (1992) 853. S. Sharpe, Phys. Rev. D46 (1992) 3146.

14. S. Aoki, et.al., Phys. Rev. Lett. 84 (2000) 238.

15. B. Bardeen, et.al., hep-lat/0007010.

16. B. Bardeen, et.al., hep-lat/0106008.

17. S. Dong, et.al., hep-lat/0108020.

18. C. Bernard, et.al., Phys. Rev. D64 (2001) 054506 .

19. U. Sharan and M. Teper, hep-ph/9910216.

20. P. Damgaard, Nucl. Phys. B608 (2001) 162.

21. R.G. Edwards, U.M Heller, R. Narayanan, Phys. Rev. D59 (1999) 094510.

22. J. Kiskis and R. Naryanan, hep-lat/0106018.

23. M. Garcia Perez, Nucl. Phys. Proc. Suppl. 94 (2001) 27.
24. A.Di Giacomo, et.al., hep-ph/0007223.

25. F. Lenz and S. Worlen, hep-ph/0010099, A. Kalloniatis and S. Nedelko, hep-ph/0108010.

26. E. Witten, Nucl. Phys. B156 (1979) 269.

27. I. Horvath, et.al., hep-lat/0102003.

28. T. DeGrand and A. Hasenfratz, heplat/0103002. I. Hip, et.al., hep-lat/0105001. T. Blum, et.al., hep-lat/0105006. G. Gattringer, et.al., hep-let/0107016. S. Dong, et.al., hep-lat/0110037.

29. R.G. Edwards and U.M Heller, heplat/0105004.

30. B. Berg, et.al., Phys. Lett. B514 (2001) 97.

31. B. Lucini and M. Teper, JHEP 0106 (2001) 050 .

32. R. Johnson and M. Teper, hep-ph/0012287.

33. U. Wenger, M. Teper, N. Cundy, these proceedings.

34. M. Teper, Z. Phys. C5 (1980) 233.

35. T. DeGrand and A. Hasenfratz, Phys. Rev. D64 (2001) 034512.

36. T. DeGrand, hep-lat/0106001.

37. M.C.Chu, et.al., Phys.Rev.D49 (1994) 6039.

38. R. Gavai, et.al., hep-lat/0107022.

39. R.G. Edwards, U.M Heller, R. Narayanan, Phys. Rev. D60 (1999) 034502.

40. Gattringer, et.al., hep-lat/0105023; Göckeler, et.al., hep-lat/0103021.

41. P. Vranas, Nucl. Phys. Proc. Suppl. 94, 177 (2001).

42. P. Hernandez, hep-lat/0110218.

43. S. Aoki, et.al., hep-lat/0110126.

44. R.G. Edwards and U.M. Heller, Phys. Rev. D63 (2001) 094505.

45. H. Neuberger, Phys. Rev. D61 (2000) 085015.

46. R. Edwards, et.al., Phys. Rev. D60 (1999) 034502. F. Berruto, et.al., Phys. Lett. B489 (2000) 243.

47. K. Orginos, et.al., hep-lat/0110074.

48. F. Berrutto, et.al., Phys.Rev.D64 (2001) 114504. M. Golterman, Y. Shamir, JHEP 0009:006,2000. I. Ichinose and K. Nagao, Nucl. Phys. B596 (2001) 231. 